\title{
Trivium
}

Revue franco-allemande de sciences humaines et sociales - Deutsch-französische Zeitschrift für Geistesund Sozialwissenschaften

$17 \mid 2014$

Relire Marcel Mauss

\section{Die Stellung von Marcel Mauss in der internationalen Anthropologie und im Gedächtnis der französischen Soziologie}

\section{Éric Brian et Florence Weber}

Traducteur : Horst Brühmann

\section{OpenEdition}

Journals

Édition électronique

URL : http://journals.openedition.org/trivium/4914

ISSN : 1963-1820

\section{Éditeur}

Les éditions de la Maison des sciences de l'Homme

Référence électronique

Éric Brian und Florence Weber, « Die Stellung von Marcel Mauss in der internationalen Anthropologie und im Gedächtnis der französischen Soziologie », Trivium [Online], 17 | 2014, online erschienen am 30 September 2014, abgerufen am 08 September 2020. URL : http://journals.openedition.org/trivium/ 4914

Ce document a été généré automatiquement le 8 septembre 2020.

\section{c) ()ㅇㅇ}

Les contenus des la revue Trivium sont mis à disposition selon les termes de la Licence Creative Commons Attribution - Pas d'Utilisation Commerciale - Pas de Modification 4.0 International. 


\title{
Die Stellung von Marcel Mauss in der internationalen Anthropologie und im Gedächtnis der französischen Soziologie
}

\author{
Éric Brian et Florence Weber
}

Traduction : Horst Brühmann

Die Rolle, die Marcel Mauss bei der Begründung einer Sozial- und Kulturanthropologie weltweit gespielt hat, ist kaum zu überschätzen. Vom Essai sur la nature et la fonction du sacrifice ${ }^{1}$, den er 1899 zusammen mit Henri Hubert veröffentlichte, bis zum Essai sur le don, der 1924 erschien, und noch darüber hinaus war Marcel Mauss die treibende Kraft der von Émile Durkheim begründeten französischen Schule der Soziologie in sämtlichen Bereichen der Religionssoziologie und Sozialanthropologie. Sein Werk stellt die Verbindung her zur englischen anthropologischen Schule, zu den großen ethnographischen Expeditionen gegen Ende des neunzehnten Jahrhunderts, die er bis 1913 ausgiebig erörterte, und zu den Forschungen Boas' über den Potlatsch und Malinowskis über den Kula, Forschungen, die er im Essai sur le don auf originelle Weise verknüpfte. Das ist auch der Grund dafür, dass Mauss in den englischsprachigen intellektuellen Kreisen, die ihm offenbar sehr nahestanden, sofort rezipiert und übersetzt wurde. ${ }^{2}$

\section{Der Preis für den Erfolg der Durkheimschen Lehre in Frankreich}

2 Mauss' Treue zu dem ursprünglichen Unternehmen, zu seinem Erbe, gab in Frankreich seit den dreißiger Jahren Anlass zu verschiedenen Vereinnahmungsversuchen. Noch heute fällt es schwer, sich von den Um- und Abwegen fernzuhalten, die die Durkheimsche Sozialwissenschaft während des zwanzigsten Jahrhunderts im kollektiven Gedächtnis genommen hat. Eine systematische Untersuchung der 
Schicksale der Durkheimschen Theorie im kollektiven Gedächtnis steht noch aus, und die Hindernisse, die sie uns hinterlassen haben, bestehen fort. So stand zum Beispiel eine erste Lektüre des Essai sur le don in dem von Georges Bataille gegründeten Collège de sociologie im Jahr 1937 im Zeichen eines pessimistischen Blicks auf die Menschheit, für den die Gabe notwendigerweise einen agonalen Charakter besitzt, zumal in modernen Gesellschaften. Nach Kriegsende berief sich hingegen die gesamte französische Anthropologie auf Mauss, um Theorien und Praktiken zu rechtfertigen, die ansonsten nichts miteinander verband.

3 So diente 1947 Denise Paulmes Veröffentlichung der Notizen, die sie sich in den zwanziger Jahren während der Vorlesungen von Mauss gemacht hatte, unter dem Titel Manuel d'ethnographie als theoretische Rückversicherung für gewisse französische Praktiken der Ethnographie, insbesondere im kolonialen Umfeld, die den zeitgleichen Entwicklungen in der amerikanischen und englischen Tradition sehr fernstanden. Die Übersetzung des Artikels von Hubert und Mauss von 1904, L'origine des pouvoirs magiques dans les sociétés australiennes, an dieser Stelle wird einen besseren Eindruck davon vermitteln können, wie die Autoren die von anderen gelieferten ethnographischen Dokumente bearbeiteten, nämlich auf eine zugleich präzise und fundierte Weise, die der historischen Quellenkritik nahesteht.

In völligem Widerspruch zu der Auffassung, die Mauss zum Vater der französischen Ethnographie erhebt, wird er in der berühmten »Einleitung " von Claude Lévi-Strauss zu der Sammlung Sociologie et anthropologie aus dem Jahr $1950 \mathrm{zu}$ einem Vorläufer der strukturalen Anthropologie. Sie teilt sein Werk in zwei Hälften und schafft damit den Mythos eines jungen Mauss, der von Durkheim abhängig ist, und stellt ihn einem reifen Mauss gegenüber, der den Weg zur strukturalen Anthropologie bahnt. Schon die Daten hätten diese allzu bequeme Lesart verbieten müssen: Die von Lévi-Strauss markierte Grenze verläuft zwischen einem Text von 1899, dem Essai sur la nature et la fonction du sacrifice, in dem Mauss und Hubert angeblich nur die Durkheimsche Analyse der kollektiven Institutionen fortführen, und einem anderen von 1902-1903, dem Essai sur la théorie de la magie ${ }^{3}$, in dem die gleichen Autoren bei ihrer Analyse des Magiers als Individuum ihre Unabhängigkeit erwiesen hätten.

Doch diese Trennung der Studie über das Opfer von der über die Magie verleitete den Herausgeber dazu, die Vorbemerkung Huberts und Mauss' zu ihrem Text in den Anhang zu verschieben. In dieser Vorbemerkung geben die Autoren die Gründe dafür an, weshalb die Durkheim-Schule von der Untersuchung des Opfers, einer kollektiven Institution par excellence, zur Erforschung der individuellen Magie überging. Hubert und Mauss ging es in Wirklichkeit darum, zu einem umfassenden Begriff der Riten im Allgemeinen zu gelangen und insbesondere zu zeigen, »in welcher Weise das isolierte Individuum in der Magie auf der Grundlage sozialer Phänomene handelt «. ${ }^{4}$ Die Geste Lévi-Strauss' zielte hingegen auf etwas ganz anderes: nämlich auf eine Trennung zwischen Mauss' soziologischen Nachfolgern, die von nun an gleichsam ins Vergessen gestürzt wurden - wie es mit Georges Gurvitch, ihrem führenden Kopf, geschah -, und neuen anthropologischen Nachfolgern, die nun abrupt sein Erbe antraten. 


\section{Vom Nutzen der internationalen Verbreitung des Werkes von Marcel Mauss}

6 Der englischsprachige Leser ist heute gewiss besser in der Lage, wieder an jenen ganz wesentlichen Gedankenfaden im Mauss'schen Werk anzuknüpfen, der das kollektive Opfer mit der individuellen Magie, aber auch das Gebet an die Götter mit der Gabe unter den Menschen verbindet. Dafür waren Übersetzungen nötig! Und da sich die größten englischen Anthropologen in den Dienst dieses Unternehmens stellten, verfügt der anglophone Leser inzwischen über mehrere verschiedene, ausführlich kommentierte Bände: mehrere Ausgaben von The Gift (mit einem Vorwort von EvansPritchard und einem späteren von Mary Douglas), Sacrifice (eingeleitet von EvansPritchard), A General Theory of Magic, seit kurzem aber auch On Prayer (2004). So wurde auf der anderen Seite des Kanals und des Atlantiks die Arbeit von Mauss nicht von der Rezeption der Durkheim-Schule getrennt. Die Namen seiner frühen Mitarbeiter, Henri Hubert und Robert Hertz, sind dort mit Recht fast ebenso bekannt wie der von Mauss. Die Verbindungen zwischen Religionssoziologie und Ethnographie werden besser verstanden und ebenso Mauss' uneingeschränkte Zugehörigkeit zur Durkheim-Schule.

7 In Frankreich kommt Victor Karady das Verdienst zu, 1968 den Versuch unternommen $\mathrm{zu}$ haben, den damaligen Studenten ein vollständigeres und insofern zutreffenderes Bild des Werkes von Mauss und seiner Verbindungen mit dem Gemeinschaftsunternehmen der Année sociologique zu bieten. Die von Karady begonnenen und fortgeführten Arbeiten kamen dem Versuch einer Neubegründung der von Durkheim inspirierten französischen Soziologie durch Pierre Bourdieu sehr gelegen. Ebenso wie für Mauss selbst waren für Bourdieu Soziologie und Anthropologie unzertrennlich. Doch bei der Durchführung dieser gelehrten Arbeit geriet Karady in ein editoriales Minenfeld: Die Texte der Sammlung Sociologie et anthropologie konnten in die Euvres nicht aufgenommen werden, und die Veröffentlichung der Essais de sociologie ohne kritische Einleitung oder Präsentation bot zwar einen unmittelbaren Zugang zu grundlegenden Texten, gab aber dem Werk das Bild eines Kaleidoskops und ließ seine Chronologie verschwimmen. Seither berufen sich mehrere Traditionen auf Mauss und bilden ebenso viele Strömungen berechtigter oder vermeintlicher Erben. Es ist hier nicht der Ort, über die Legitimität der einen oder der anderen ein Urteil zu fällen. Allenfalls kann man sagen, dass bisher von ihnen nichts ausging, das sie ihrer Wiedervereinigung unter der Ägide des berühmten Neffen näher gebracht hätte.

8 So konnte sich auch die Erinnerung an die Durkheimsche Soziologie den allgemeinen Regeln nicht entziehen, die über das Schicksal spezialisierter Formen des Gedächtnisses herrschen, Regeln, die für die Überlieferung besonderer, nicht nur wissenschaftlicher Kenntnisse eigentümlich sind. Diese Dynamiken, die Maurice Halbwachs so gut nachzuzeichnen verstand, bestehen aus topischen Revisionen (in diesem Fall Neubesetzungen von Forschungsgebieten) und der Umarbeitung von Traditionen (einige Beispiele dafür haben wir eben gesehen), mit einem Wort, aus ständigen Aushandlungsprozessen zwischen der Gegenwart der Disziplinen, den Spuren ihrer Vergangenheit, selbst der jüngsten, und den vorläufigen, von Zeit zu Zeit verkündeten Programmen für ihre Zukunft. Wie es scheint, waren die neunziger Jahre in Frankreich für eine Neubewertung des Mauss'schen Werkes eher günstig, auch wenn sie auf Spezialgebiete beschränkt blieb, die als allzu abgeschottet galten. Die Konsolidierung der sozialwissenschaftlichen Disziplinen in den universitären Ausbildungsgängen 
begann in Frankreich 1958 mit den Studienabschlüssen der maitrise und des anschließenden Doktorats in den jeweiligen neuen Fächern, die sich von der Philosophie, aus der sie hervorgegangen waren, getrennt hatten. Insgesamt weist diese Entwicklung gewisse Analogien mit der Herausbildung der Naturwissenschaften im Deutschland des neunzehnten Jahrhunderts auf, als sich diese Disziplinen vom Hauptast der Naturphilosophie, die bis dahin unter dem Titel Philosophie gelehrt worden war, losrissen.

Dieser rasche Vergleich bedürfte zahlreicher näherer Untersuchungen zur Dynamik der disziplinären Beziehungen in den Natur- und Geisteswissenschaften über einen langen Zeitraum, der sich vom siebzehnten bis zum zwanzigsten Jahrhundert erstrecken müsste und der je nach der bevorzugten Wissenschaftssprache anders zu behandeln wäre. Der Essai sur le don bot Anlass zu einer anthropologischen Neulektüre, die zwei grundlegende Punkte herausstellte: einerseits die "Kraft der Dinge« (Bazin, übersetzter Text) und andererseits die Unterscheidung zwischen "Transaktion« und "Übertragung" (Weber, übersetzter Text; Testart [2007]). Camille Tarot wiederum ( übersetzter Text) stellt die Gedankenlinie in den Vordergrund, die Marcel Mauss vom Opfer und von der Magie zum Gebet und dann zur Gabe führte, während Bruno Karsenti (übersetzter Text) den von Claude Lévi-Strauss gebahnten Weg weiterverfolgt. Es war dies ein Weg, der Mauss zu einem widerspenstigen Erben Durkheims machte, was ihn in den philosophischen und anthropologischen Milieus Frankreichs - die, wie oft bemerkt, traditionell eng zusammenhängen - akzeptabler als seinen Onkel erscheinen ließ.

\section{Die politischen Einsätze des Essai sur le don}

Doch an dieser Stelle der Analyse ist zu beachten, dass es sowohl in der französischen wie in der deutschen Tradition in Wirklichkeit politische Fragen sind, die einer versöhnlichen Lektüre des Werkes Durkheims und der Durkheimianer entgegenstehen; einer Lektüre, die in England und den USA viel leichter möglich ist, weil dort solche Aspekte weitgehend übersehen werden. Auch wenn es nicht darum gehen kann, das Mauss'sche Werk auf den Essai sur le don zu reduzieren, wird doch in diesem Text - wie Marcel Fournier gezeigt hat ${ }^{5}$ - die Verbindung zwischen Mauss' politischen und akademischen Aktivitäten hergestellt. Und es ist gewiss kein Zufall, dass das Interesse an diesem Text genau in dem Moment wiedererwacht ist, in dem der »kontinentaleuropäische« Wohlfahrtsstaat ${ }^{6}$ heftig in Frage gestellt wurde.

\section{Bruch mit dem Bolschewismus und mit dem Faschismus}

11 Mauss hat den Essai sur le don genau zu der Zeit verfasst, als er sich um eine - nach eigenen Worten - "soziologische Beurteilung" des russischen Bolschewismus und des italienischen Faschismus bemühte; genau zu der Zeit, als er über die Verbindungen zwischen den Nationen, die Arbeitsteilung zwischen den Nationen und den Internationalismus reflektierte. In den Schlussfolgerungen des Essai findet man einen Verweis auf den Mythos König Artus' und seiner Tafelrunde, eine Metapher für den Völkerbund, in den die Intellektuellen der zwanziger Jahre große Hoffnungen setzten. Als sozialistischer Aktivist und Bewunderer von Jean Jaurès bemühte sich Mauss, eine sozialistische Partei aktiv zu unterstützen, die noch von der Spaltung geschwächt war, 
aus der 1920 die französische kommunistische Partei hervorgegangen war. Der Essai trägt die Spur dieser Suche nach politischen Lösungen, die mit der Durkheimschen Idee intermediärer Kollektive zwischen Individuum und Staat brechen. Mauss fürchtete in der Tat, Merkmale dieser intermediären Kollektive in den russischen Sowjets und in den italienischen faschistischen Organisationen wiederzufinden.

Eine Furcht, die aus der Rückschau umso begründeter erscheint, als im Verlauf eines oder zweier Jahrzehnte einige überzeugte Durkheimianer, mit denen Mauss in enger Beziehung stand, sich in Bewegungen der extremen Rechten engagierten, die aus der sozialistischen Bewegung hervorgegangen waren, etwa Marcel Déat, auf den wir zurückkommen werden. Es wäre einmal systematisch zu untersuchen, inwieweit und wie sich die Philosophie, die Anthropologie und die Soziologie der Jahre von 1930 bis 1960 auf Durkheim und die Durkheimianer berufen haben - in mindestens drei Sprachen, Französisch, Deutsch und Englisch -, um zu ermessen, welchen Einfluss nach dem Krieg die von den einen oder anderen vor dem Krieg vertretenen Positionen besaßen.

\section{Bruch mit der Mildtätigkeit}

Es ist nicht übertrieben zu sagen, dass über die implizite Kritik an der ökonomischen Theorie des Tausches hinaus eine ganz explizite Kritik des Almosens den zentralen politischen Einsatz des Essai sur le don ausmacht. Das Almosen ist genau der Typus der Gabe ohne Rückgabe, der die Armen erniedrigt, sobald sie es ohne die Möglichkeit einer Erwiderung annehmen. «Milde Gaben verletzen den, der sie empfängt, und alle unsere moralischen Bemühungen zielen darauf $a b$, die unbewußte schimpfliche Gönnerhaftigkeit des reichen >Almosengebers` $\mathrm{zu}$ vermeiden. ${ }^{7}$ Will man die Sozialpolitik von ihrer Herkunft aus der Mildtätigkeit lösen, um sie annehmbar und nicht erniedrigend zu machen, ist zunächst einmal ein Bedeutungswandel erforderlich. Die begriffliche Anstrengung von Mauss zielt darauf, die damals entstehende Sozialpolitik nicht als Gabe darzustellen, die den Armen erwiesen wird, sondern als Gegengabe, die die Arbeiter im Austausch für die ursprüngliche Gabe erhalten, die sie mit ihrer Arbeit geliefert haben und für die der Lohn keine hinreichende Gegengabe darstellt. Weder die Dienstherrn noch die Gesellschaft, sagt Mauss, sind mit der Zahlung eines Lohns ihnen gegenüber quitt (»aller Schuld [...] enthoben «8). Heute würde man von einem strukturellen Defizit des Arbeitsvertrags sprechen.

Was Mauss jedoch voraussieht und was erst nach 1945 wirklich eintreten wird, ist eine Entwicklung, die die soziale Schuld der Unternehmen auf die Gesellschaft insgesamt verschiebt, von der unternehmerischen Gönnerhaftigkeit (dem reichen "Almosengeber«) zur Anonymität des Kollektivs, von der persönlichen Gabe, die zur mechanischen Solidarität gehört, zur anonymen Gabe, die zur organischen Solidarität rechnet. ${ }^{9}$ Diese Entwicklung, jahrzehntelang vorbereitet durch die Annäherung zwischen der Arbeiterbewegung, den Intellektuellen und der philanthropischen Bourgeoisie, wird erst in den Anstrengungen zur Schaffung der Sozialversicherungen unmittelbar nach der Befreiung 1945 ihre volle Entfaltung finden. Heute jedoch, zwanzig Jahre nachdem damit begonnen wurde, den Wohlfahrtsstaat ideologisch in Frage zu stellen, konnte die Politik der sozialen Fürsorge die Gefahr einer Regression auf Wohltätigkeit nicht vermeiden: In mehr oder weniger ausgeklügelten Formen kettet diese Politik die permanenten Gabenempfänger (die "Sozialhilfeempfänger«, die 
Armen) an eine permanente Schuld. Was heute fehlt, sind nicht so sehr die finanziellen Mittel als eine Bewegung, die es erneut verstünde, politische Aktivisten, Intellektuelle und Philanthropen zusammenzuschließen, um aus der Ideologie der Nähe, der persönlichen Beziehung und der individualisierten Behandlung der sozialen Frage herauszukommen und das stark geschwächte Konzept "sozialer Rechte» wiederzuentdecken.

Um mit der Wohltätigkeit zu brechen, hatte Mauss sich entschieden, den Akzent auf die »ursprüngliche Gabe« zu legen, die einer als Gegenleistung gedachten Sozialpolitik entsprach. Es können mehrere andere Pfade beschritten werden, um die persönliche Herrschaftsbeziehung zwischen dem Philanthropen und »seinen« Armen zu beenden. Man kann die Betonung auf eine Vergemeinschaftung der Ressourcen innerhalb einer Gruppe legen, zum Beispiel der häuslichen Gruppe: Ergeben sich die Konflikte zum Zeitpunkt einer Scheidung nicht gerade aus der Tatsache, dass die Spirale ihren unbestimmten zeitlichen Horizont verliert und dass man, gegenwärtig jedenfalls, "abzurechnen" gezwungen ist, die Vergangenheit im Lichte der Auflösung der Gruppe lesen muss? Man kann versuchen, eine nicht-demütigende Generosität zu erweisen: das ist der Versuch Luc Boltanskis, eine spezifische Haltung zu definieren, die Agape, eine einzigartige, nicht-berechnende Liebe. Eine andere Position legt den Akzent auf die Anonymität der Geber. Wenn der Gabenempfänger die Identität des Gebers nicht kennt, wie könnte er dann unfreiwillig in eine ungleiche persönliche Beziehung verwickelt werden? Man könnte diese Anonymität als das Prinzip der Umverteilung durch die Steuer betrachten. Manche der Bioethik verpflichtete Institutionen bewahren das Prinzip der Gabe ohne Gegengabe, der Blut- oder Organspende, die auf der ausdrücklichen Anonymität der Spender beruht (die dann auch nicht mehr Geber, sondern Spender heißen), so dass nichts die reine Großzügigkeit trüben könnte, die sie bezeugen, und zugleich so, dass die immense, unauslöschliche Schuld, die der Empfänger gegenüber dem Spender eingegangen ist, ihn nicht verpflichtet, in eine persönliche Beziehung unendlicher Abhängigkeit zu treten.

\section{Schaffung einer Sozialversicherung}

Der Essai stellt somit einen Wendepunkt im Verhältnis der Durkheimschen Soziologie zur Politik dar. Wenn das Werk Durkheims als eine der geistigen Grundlagen der Dritten Republik erscheinen konnte, so bezeichnet der Essai sur le don einen wesentlichen Schritt auf dem Weg zu einer Sozialversicherung à la française. Mit der Annahme, die Gesellschaft sei gegenüber den Arbeitern, die ihr ihr Leben gegeben haben, noch nicht aller Schuld enthoben, wenn sie einmal ihren Lohn empfangen haben, mit der Annahme, dass sie ihnen noch die Mittel für ein anständiges Leben im Alter und in Zeiten der Arbeitslosigkeit schulde, bricht Mauss mit den Prinzipien der Wohltätigkeit, die damals der Sozialpolitik zugrunde lagen, mit der »unbewußte[n] schimpfliche[n] Gönnerhaftigkeit des reichen >Almosengebers"«, die er in den Schlussfolgerungen seines Essai evoziert. Er bahnt den Weg zu der Idee sozialer Leistungen, die nicht mehr Almosen sind, sondern als Rechte konzipiert werden, die einen Anspruch von Individuen gegenüber der Gesellschaft als ganzer begründen.

Die Arbeit von Mauss hat sich nicht in den Fallstricken der großen Spaltung zwischen den abendländischen Gesellschaften (»wir») und dem Rest der Welt (»sie«) verfangen, wie uns eine anthropologische Disziplin weismachen wollte, die vor den 
zeitgenössischen Gesellschaften allzu lange ängstlich zurückscheute. Ganz im Gegenteil werden seine Analysen der Gabe in den ursprünglichen und archaischen Gesellschaften von dem Anspruch getragen, Antworten auf »einige Probleme« zu finden, »die die Krise unseres Rechts und die Krise unserer Wirtschaft stellen« - und wir ergänzen: zu einem Zeitpunkt, zu dem extreme Lösungen, der Bolschewismus in Russland seit 1917 und der Faschismus in Italien seit 1922, den Sieg über eine vernünftige Reform des marktwirtschaftlichen Kapitalismus davongetragen hatten, eine Reform, die es erlaubt, die sozialen Ungleichheiten zu korrigieren und die wirtschaftlichen Krisen, die er erzeugt, zu verhüten. Zwanzig Jahre, ein Weltkrieg und ein Bürgerkrieg waren in Frankreich noch notwendig, bis sich die Mauss'schen Intuitionen in der Form eines kohärenten Sozialmodells verwirklichen sollten, das die Lohnarbeit in den Mittelpunkt der sozialen Solidarität stellt, als eine Gabe, die einen Ausgleich über den bloßen Lohn hinaus fordert. Sechzig Jahre später haben die Transformationen der Lohnarbeitsgesellschaft dieses europäische Modell, das man konservativ oder kontinentaleuropäisch nennt, in Gefahr gebracht. Ein Grund mehr, den Essai sur le don noch einmal zu lesen.

Es geht hier nicht um Kleinigkeiten. Mit seiner Untersuchung des Geschenkeaustauschs leistet Mauss eine theoretische Kritik an der naturalistischen Konzeption des Marktes und eine politische Kritik an der Konzeption der sozialen Fürsorge als Wohltätigkeit in einer Zeit, die von der Erschütterung des Konsenses zwischen Soziologen, Philanthropen und Arbeiterbewegung gekennzeichnet war, eines Konsenses, der um die Wende zum zwanzigsten Jahrhundert die Entstehung der ersten Sozialgesetze ermöglicht hatte.

Vor dem Essai sur le don waren solche nicht-tauschförmigen Leistungen zwar bereits untersucht, aber noch nicht als wissenschaftliches Objekt konstituiert worden. Der Essai sur le don enthält also eine kritische Ergänzung der klassischen Analysen der Marktwirtschaft: eine Analyse der Ökonomie der Gabe, wenn man den Akzent auf ihre Einheit legen möchte, oder der Ökonomien ohne Tausch, wenn man ihre Heterogenität in den Vordergrund stellen möchte.

\section{Den Schrecken des sozialen Gedächtnisses der Sozialwissenschaft überwinden}

Die soziale Frage und die Metamorphosen ihrer Lösung im zwanzigsten Jahrhundert drängen sich unmittelbar auf, sobald man ernsthaft mit einer Mauss-Lektüre beginnt. Freilich weist das zwanzigste Jahrhundert, wiederum unter dem Gesichtspunkt des kollektiven Gedächtnisses der französischsprachigen Anthropologie und Soziologie, eine weitere Besonderheit auf, die hier im Hinblick auf die Durkheimsche Soziologie hervorzuheben ist. Es handelt sich um den Konflikt zwischen dem eigentlich wissenschaftlichen Gedächtnis, das für die direkte oder kritische, verkürzte oder umfassendere Weitergabe der Wissensbestände einer Disziplin notwendig ist, und dem politischen Gedächtnis eines desaströsen Jahrhunderts, in dessen Verlauf riesige Staaten und Gesellschaften untergegangen sind und militärische wie politische Greueltaten begangen wurden, oftmals unter dem Deckmantel pseudogelehrter Rationalisierungen, $z u$ denen die Sozialwissenschaften herangezogen wurden. Schon 1936, als das Schlimmste noch bevorstand, wagte der Historiker Lucien Febvre in seiner Einleitung zum siebten Band der Encyclopédie française - der eine Bilanz der Erkenntnis 
des Menschen ziehen und der politischen Instrumentalisierung der Humanwissenschaften entgegenwirken wollte - das Wort: „Der Feind, das ist die Übervereinfachung! « Es ist gewiss von Belang, dem heutigen, mit der Pariser Szene der Sozialwissenschaften in den späten dreißiger Jahren wenig vertrauten Leser zu erklären, dass das Centre de documentation sociale der École normale supérieure in der Rue d'Ulm - Hochburg der Durkheimianer und der Sozialisten - unter den wohlwollenden Augen des Direktors der École, Célestin Bouglé, in den Vorkriegsjahren von einem sehr engagierten agrégé betreut wurde, nämlich von dem bereits oben erwähnten Marcel Déat - einem brillanten Schüler der ENS, der wie kein anderer dafür designiert war, in den fünfziger und sechziger Jahren das aktuellste Wissen an der Universität zu lehren. Déat war in der Zeit zwischen den Kriegen auf der politischen Bühne tätig (als Aktivist, Vordenker politischer Strömungen innerhalb der SFIO, Gründer politischer Parteien, Abgeordneter, Minister). Er formulierte Denkrichtungen, die sich als innovativ ausgaben, und zog verworrene Querverbindungen vom Sozialismus zum Faschismus und zum Antibolschewismus der Nazis, während er sich vor dem Krieg gelegentlich als Antifaschist darstellte. Ohne uns bei der Analyse dieses erstaunlichen und zwielichtigen Weges aufzuhalten, wollen wir uns auf den Hinweis beschränken, dass die Erinnerung daran wie eine politische Leiche im Keller der Durkheimschen Soziologie vergraben blieb. Drei aufschlussreiche Orientierungspunkte seien erwähnt. Zunächst ein Buch: "La sociologie«, erschienen bei Alcan in der kleinen Reihe "Notions de philosophie» und zwischen 1925 und 1939 mehrfach wiederaufgelegt, damals ein Standardwerk der Durkheimschen Soziologie. Kein Philosoph, zumal an der École normale unter Bouglé, und kein aufgeweckter Student kam seinerzeit an diesem Buch vorbei. Zweitens ein aufsehenerregender Artikel, der in L'Euvre erschien, einer agitatorischen, zu dieser Zeit pazifistischen Tageszeitung, in der Déat als Leitartikler die Feder führte: »Sterben für Danzig?«, am 4. Mai 1939, als sich der Krieg ankündigte. Dieser Weg, auf dem sich philosophische Überzeugungen, politische Rechtfertigungen und flammende politische Proklamationen verflochten, führte Marcel Déat schließlich dahin, ab März 1944 in der kollaborationistischsten Regierung, die Frankreich je hatte, die Aufgaben eines Ministers für Arbeit und nationale Solidarität zu übernehmen, und zwar, was seine Person betraf, auf ausdrücklichen Wunsch der deutschen Besatzer. Nach der Befreiung Frankreichs folgte er sogar mit Pétain und Laval seinen Herren und Meistern nach Sigmaringen, um schließlich in irgendeinem behaglichen italienischen Kloster unterzutauchen. Im politischen Gedächtnis verbindet sich mit Déat, dass er im Juni 1945 nach der Befreiung in Abwesenheit zum Tode verurteilt wurde.

21 Doch wie steht es mit dem sozialen Gedächtnis, um den von Halbwachs geprägten Begriff aufzunehmen, diesem Produkt der spannungsgeladenen Zusammenführung der aktuellen spezialisierten Erinnerungsschichten einer jeweiligen Epoche? Es ist geprägt durch eine Aporie zwischen der allen Zeitgenossen bekannten Tatsache eines politischen Abwegs, der umso verwerflicher ist, als es der eines jungen Dozenten ist, der im philosophischen Spektrum der Vorkriegszeit seinen anerkannten Platz hatte, eines Politikers, der bis zum Äußersten gegangen war in dem, was zur Zeit der Befreiung als Landesverrat auch nur denkbar war, bis zum äußersten Verrat an einem geistigen Modell, das von einer der angesehensten Lehrinstitutionen getragen wurde und jener anderen, seit den vierziger Jahren ausgelöschten Tatsache, dass diese Person in der Zwischenkriegszeit in Frankreich der maßgebliche Lehrer der Durkheimschen Soziologie war. Kein Zweifel, dass die agrégés, die in der unmittelbaren Nachkriegszeit 
und danach tätig waren, oder dass diejenigen, die am Vorabend des Krieges 1939 ihre Ausbildung erhalten hatten, sich diese Dinge nicht vergegenwärtigen konnten, ohne beim Erinnern jenen Schrecken zu verspüren, den andere nach dem Krieg an so vielen anderen Orten empfunden haben. Mit dem Kalten Krieg legte sich eine weitere undurchsichtige Schicht darüber, die die relevanten intellektuellen Abhängigkeiten zwischen den exemplarischen Durkheimianern verschwimmen ließ, Durkheim natürlich, Mauss, Simiand, Halbwachs, Hertz und Hubert, um nur die wichtigsten anzuführen, die unser Gedächtnis dieser Wissenschaft wenigstens noch bewahrt hat.

Es bleibt den Historikern und den Trägern des wissenschaftlichen Gedächtnisses der Vorkriegszeit vorbehalten, ein klares und konstruktives Bild unserer intellektuellen Verpflichtung gegenüber der Durkheim-Schule und ganz besonders gegenüber dem Werk von Marcel Mauss zusammenzusetzen - die eine wie das andere Opfer der Bedingungen, unter denen das Gedächtnis der Durkheimschen Wissenschaft nach so vielen Umarbeitungen, Lücken und interessegeleiteten temporären Umbesetzungen uns heute zugänglich ist. Wir müssen dieses neue Unternehmen beginnen, um zu diesen fast hundert Jahre alten Texten Zugang zu finden und um sie sinnvoll einordnen zu können. Zwischen ihrer Niederschrift und unserer Lektüre liegen so viel Schrecken, gewiss. Um uns von den Verwerfungen und dem Unverständnis zu lösen, die von den in der zweiten Hälfte des zwanzigsten Jahrhunderts herrschenden Lehren genährt wurden, um die willkürlichen und überholten Revisionen des soziologischen Gedächtnisses zu unterlaufen, verfügen wir über ein Mittel, dem unsere Vorgänger misstraut hätten: Wir können die internationale Verbreitung von Übersetzungen fördern, die Konfrontation der Deutungen und der kritischen Analysen, damit sich, widerständiger denn je, das internationale Gedächtnis der Sozialwissenschaften herausbildet.

\section{BIBLIOGRAPHIE}

Brian, E. (2008): »Portée du lexique halbwachsien de la mémoire«, in: M. Halbwachs, La Topographie légendaire des évangiles en Terre sainte. Étude de mémoire collective, neu hg. von M. Jaisson, Paris, PUF, S. 113-146.

Esping-Andersen, G. (1990): Les trois mondes de l'État-providence, Paris, PUF.

James, W. / Allen, N. J. (1998): Marcel Mauss. A Centenary Tribute, Oxford/New York, Berghahn Books.

Mauss, M. (1947): Manuel d'ethnographie, nach Vorlesungsnotizen aufgezeichnet von D. Paulme, Paris, Payot [deutsch (2013): Handbuch der Ethnographie, aus dem Französischen von L. Dinkel und A. Haarmann, Paderborn, Fink].

Mauss, M. (1950): Sociologie et anthropologie, mit einer Einleitung in das Werk von M. Mauss von C. Lévi-Strauss, Paris, PUF 1950, 3. verm. Aufl. 1966 [deutsch (1974): Soziologie und Anthropologie, 2 Bde., mit einer Einleitung von C. Lévi-Strauss, übersetzt von E. Moldenhauer und H. Ritter, München, Hanser; Nachdruck (2010): Wiesbaden, VS Verlag für Sozialwissenschaften]. 
Mauss, M. (1968-1969): CEuvres, 3 Bde., hg. von V. Karady, Paris, Minuit [daraus deutsch (2012): Schriften zur Religionssoziologie, hg. und eingeleitet von St. Moebius, F. Nungässer und Ch. Papilloud, übersetzt von E. Moldenhauer und H. Ritter, Berlin, Suhrkamp].

Mauss, M. (1974): Essais de sociologie, Paris, Seuil-Minuit.

Mauss, M. (1997): Écrits politiques, hg. und eingeleitet von M. Fournier, Paris, Fayard.

Mauss, M. (2012): Essai sur le don, mit einer Einleitung von F. Weber, Paris, PUF (= Bd. 6 der »Serie Mauss", coll. Quadrige) [deutsch (1968): Die Gabe. Form und Funktion des Austauschs in archaischen Gesellschaften, mit einem Vorwort von E. E. Evans-Pritchard, übersetzt von E. Moldenhauer, Frankfurt am Main, Suhrkamp; um einen Anhang von H. Ritter erweiterte Taschenbuchausgabe 1990].

Sigaud, L. (1996): „Le courage, la peur et la honte. Morale et économie dans les plantations sucrières du Nordeste brésilien«, Genèses, 25, S. 72-90.

Steiner, Ph. (2014): »Mauss, les groupements professionnels et »l'atmosphère du don «, Études sociales, im Erscheinen.

Testart, A. (2007): Critique du don. Études sur la circulation non marchande, Paris, Syllepse.

\section{NOTES}

1. Mauss (1968), Bd. 1, S. 193-207 [deutsch in: Mauss (2012), S. 97-216].

2. James / Allen (1998).

3. Mauss (1950) [deutsch in: Mauss (1974), Bd. 1, S. 43-179].

4. Hubert / Mauss, in: Mauss (1950), S. 140 [deutsch in: Mauss (1974), Bd. 1, S. 178].

5. Mauss (1997).

6. Esping-Andersen (1990).

7. Mauss (1950), S. 258 [deutsch: Mauss (1968), S. 157].

8. Mauss (1950), S. 260 f. [deutsch: Mauss (1968), S. 160].

9. Sigaud (1996), Steiner (2014).

\section{INDEX}

Schlüsselwörter : Soziologiegeschichte

Mots-clés : Histoire de la sociologie

\section{AUTEURS}

\section{ÉRIC BRIAN}

Éric Brian ist directeur d'études an der École des hautes études en sciences sociales (EHESS). Nähere Informationen finden Sie hier. 


\section{FLORENCE WEBER}

Florence Weber ist Professorin an der École normale supérieure (ENS). Nähere Informationen finden Sie hier. 